\title{
Efficacy of ketoprofen for treatment of spontaneous, culture-negative, mild cases of clinical mastitis: A randomized, controlled superiority trial
}

\author{
G. S. Latosinski, M. J. Amzalak, and J. C. F. Pantoja* (D) \\ Department of Veterinary Hygiene and Public Health, College of Veterinary Medicine and Animal Science, Sao Paulo State University (UNESP), \\ Botucatu, Sao Paulo 18618-681, Brazil
}

\begin{abstract}
The objective of this randomized, controlled superiority trial was to assess the efficacy of ketoprofen for the treatment of spontaneous, culture-negative clinical mastitis cases that were not treated with antimicrobials. Holstein cows from 3 herds were eligible for inclusion if they had mild or moderate culture-negative clinical mastitis cases in 1 quarter or more. Upon detection of clinical mastitis, farm personnel performed on-farm culture (OFC) using commercially available bi-plates. Samples used for OFC were also cultured in a research laboratory. Cows with culture-negative clinical mastitis that met the inclusion criteria were randomly allocated to 1 of 2 experimental groups: in the ketoprofen (KET) group, cows received an intramuscular injection of 3 $\mathrm{mg} / \mathrm{kg}$ of ketoprofen upon confirmation of a negative OFC result; and in the control (CON) group, cows received no treatment or placebo. Milk samples were collected 14 and $21 \mathrm{~d}$ after detection of clinical mastitis for microbiological examination and somatic cell counting. Study outcomes were clinical cure (within $7 \mathrm{~d}$ after inclusion in the study), relapse (within $14 \mathrm{~d}$ after inclusion) and recurrence of clinical mastitis (15 to 90 $\mathrm{d}$ after inclusion), risk of new intramammary infection, and quarter milk somatic cell count at 14 and $21 \mathrm{~d}$. We used Cox proportional hazards, logistic regression, and repeated-measures models to compare each outcome between groups. After exclusion of moderate cases (n $=6)$, a total of 123 clinical mastitis cases $(\mathrm{CON}=58$ and KET $=65$ ) were used for analyses. Risks of clinical cure $[83.08 \%(54 / 65)$ and $91.23 \%(52 / 57)$; hazard ratio $=1.20,95 \%$ confidence interval $(\mathrm{CI})=0.82-1.76]$, relapse $[19.23 \%(10 / 52)$ and $18.00 \%(9 / 50)$; hazard ratio $=1.09,95 \% \mathrm{CI}=0.45-2.62]$, and recurrence of clinical mastitis $[17.31 \%(9 / 52)$ and $18.00 \%(9 / 50)$; hazard
\end{abstract}

Received August 27, 2019.

Accepted November 24, 2019.

*Corresponding author: jose.pantoja@unesp.br ratio $=1.26,95 \% \mathrm{CI}=0.49-3.38]$ were not different between the KET and CON groups, respectively. The odds of a new intramammary infection at $14 \mathrm{~d}[20.75 \%$ $(11 / 53)$ and $29.79 \%(14 / 47)$; odds ratio $=1.76,95 \%$ $\mathrm{CI}=0.66-4.73]$ or $21 \mathrm{~d}[28.57 \%(12 / 42)$ and $15.22 \%$ $(7 / 46)$; odds ratio $=0.45,95 \% \mathrm{CI}=0.16-1.30]$ were not different between the KET and CON groups, respectively. Mean somatic cell count was not different between the groups at 14 or $21 \mathrm{~d}$. The results of this study suggest that a single intramuscular injection of ketoprofen as sole treatment for OFC-negative, mild clinical mastitis did not reduce time to clinical cure, relapse or recurrence of clinical mastitis, risk of subsequent intramammary infection, or milk somatic cell count compared with untreated controls.

Key words: bovine clinical mastitis, therapy, ketoprofen

\section{INTRODUCTION}

The dairy industry is under scrutiny due to societal concerns such as animal welfare and the large-scale use of antimicrobials (Cardoso et al., 2016). Of all diseases in dairy cows, mastitis is not only the costliest (Halasa et al., 2007), but also the main reason for the use of antimicrobials (Nobrega et al., 2017; Redding et al., 2019). Of all clinical mastitis cases, 24 to $50 \%$ are negative following conventional microbiological culture of milk (McDougall et al., 2007; Lago et al., 2011a; Cortinhas et al., 2016) and do not require antimicrobial treatment (Fuenzalida and Ruegg, 2019). Antimicrobial treatment of all cases of clinical mastitis, without knowing culture results, leads to the excessive use of antimicrobials and unnecessary exposure of cows to these agents.

The advent of techniques for the rapid identification of mastitis pathogens and selective treatment of cases of clinical mastitis that can benefit from antimicrobials, such as on-farm culture (OFC), has been a keystone for rational management of clinical mastitis. Use of OFC can reduce antimicrobial usage by 50 to $68 \%$ (Lago et al., 2011a; Vasquez et al., 2017). In addition, the $24 \mathrm{~h}$ interval between case detection and treatment based on 
OFC results does not affect outcomes such as risk of clinical and bacteriological cure, recurrence, culling and death, or mean milk production and SCC (Lago et al., 2011a,b; Vasquez et al., 2017).

An opportunity explored by farmers and consultants when using $\mathrm{OFC}$ is the treatment of culture-negative clinical mastitis with non-steroidal anti-inflammatory drugs (NSAID), based on the hypothesis that time to clinical cure would be reduced. The NSAID class has anti-inflammatory, antipyretic, and analgesic action that inhibits synthesis of pro-inflammatory enzymes (Vane and Botting, 1998). A recent study has reported that NSAID were used as part of clinical mastitis therapy in $22.2 \%$ of 564 North American farms (Kayitsinga et al., 2017). Despite their frequent use, efficacy of NSAID for the treatment of spontaneous clinical mastitis is still poorly understood. Only one study has reported that use of an NSAID (meloxicam), in addition to intramammary treatment with antimicrobials (cefalexin and kanamycin), improved remission of clinical signs and risk of microbiological cure compared with antimicrobial treatment alone (McDougall et al., 2016).

Ketoprofen is an NSAID characterized by rapid absorption and elimination following intramuscular administration. Via this route, it reaches maximum plasma concentration at 30 to $60 \mathrm{~min}$, and $80 \%$ is eliminated within $12 \mathrm{~h}$ (NOAH, 2018). Ketoprofen is approved for mastitis therapy in lactating cows in countries such as Canada and Brazil. Because ketoprofen is characterized by a rapid onset of action, a short plasma half-life, low toxicity (Kantor, 1986), and no need for milk withdrawal after treatment, it could be justified for clinical mastitis therapy. Although some studies have evaluated the efficacy of ketoprofen for clinical mastitis treatment (Shpigel et al., 1994; Banting et al., 2008; Dan et al., 2018), outcomes such as risk of clinical and microbiological cure and relapse and recurrence of clinical mastitis have not been studied. To our knowledge, the efficacy of ketoprofen to treat culture-negative clinical mastitis cases following OFC results has not been studied. Use of ketoprofen in such cases could result in economic benefit to farmers and improved animal welfare.

The objective of this trial was to assess the efficacy of ketoprofen for the treatment of spontaneous, culture-negative clinical mastitis in cases that were not treated with antimicrobials following OFC. We tested the hypothesis that a single intramuscular injection of ketoprofen upon confirmation of an OFC-negative result would decrease time to clinical cure and relapse or recurrence of clinical mastitis, decrease risk of subsequent IMI, and lower milk SCC compared with untreated controls.

\section{MATERIALS AND METHODS}

This study was approved by the Sao Paulo State University Ethics Committee for Animal Use, protocol 0202/2017.

\section{Experimental Design}

We conducted a randomized, controlled superiority trial, reported according to the REFLECT statement's guidelines for the valid and ethical conduct of clinical trials including production animals (Sargeant et al., 2016).

\section{Eligibility Criteria}

We used a convenience sample of 3 dairy herds located in Sao Paulo and Minas Gerais states, Brazil. Farms had to use OFC for all cases of clinical mastitis and make a treatment decision (treat or not treat) upon completion of microbiological diagnosis. Culturenegative mammary quarters could not be treated with antimicrobials, except in severe cases that required immediate treatment. Herds had to have at least 200 lactating cows (Holsteins) and be willing to comply with the study protocol.

Quarters were eligible for inclusion if they had mild or moderate clinical mastitis (based on the severity scale proposed by Wenz et al., 2001) that was OFC-negative. If a cow presented with clinical mastitis in 1 quarter or more, quarters were eligible for inclusion if all affected quarters were OFC-negative. Quarters were not eligible if the cow had been treated with antimicrobials or antiinflammatories within $14 \mathrm{~d}$ before the case, had been previously included in the study, or presented with any disease other than clinical mastitis.

\section{Case Definition}

Clinical mastitis cases were detected by trained milking technicians, at all milkings, by visually examining the milk and the mammary gland. The severity of clinical mastitis was scored according to Wenz et al. (2001). Mild cases showed only changes in milk appearance (e.g., color or viscosity); Moderate cases showed changes in milk appearance and any sign of inflammation in the mammary gland. Severe cases showed systemic signs (such as fever, prostration, and dehydration) in addition to signs in milk and the mammary gland.

\section{Sampling Strategy and Interventions}

Initially, farms were visited to explain the study protocol and obtain experimental consent. Milking 
managers and technicians were trained to perform clinical mastitis detection, treatment, severity scoring, and aseptic collection of milk samples.

Upon detection of clinical mastitis, $20 \mathrm{~mL}$ of milk was aseptically collected from each affected quarter. Then, OFC was performed by farm personnel using commercially available bi-plates containing McConkey and blood agars (PROBAC, Sao Paulo, SP, Brazil). A sterile swab was used to inoculate milk onto the plates, which were incubated at $37^{\circ} \mathrm{C}$ for $24 \mathrm{~h}$. Milk samples used for OFC were frozen on the farms and shipped for microbiological analysis to the Sao Paulo State University Mastitis Research and Diagnostic Laboratory. On farms A and B, OFC was performed twice a day (morning and afternoon); on farm $\mathrm{C}$, $\mathrm{OFC}$ was performed daily (morning). Because the herd on farm $\mathrm{C}$ was large (2,020 lactating cows) and experienced several OFCnegative cases per day, cases were included in the study once a week, so that farm personnel could correctly follow the study protocol.

Cows with 1 OFC-negative quarter or more that met the inclusion criteria were randomly allocated to 1 of 2 experimental groups: KET, in which cows received an intramuscular injection of $3 \mathrm{mg} / \mathrm{kg}$ of ketoprofen (Ketofen; CEVA Saude Animal, Paulinia, SP, Brazil), upon confirmation of a negative OFC result; and CON, in which cows received no treatment or placebo.

\section{Randomization}

Although outcomes were analyzed at the quarter level, randomization was performed at the cow level, so that all eligible quarters of a cow were allocated to the same group. Within each herd, we used blocked randomization (Friedman et al., 2010) to form blocks of 4 cows, such that each block contained 2 cows randomly allocated to each group. This procedure was repeated until the required sample size was achieved. Each farm received a stack of previously randomized numbered envelopes, which included a field form indicating the group to which the cow was allocated and fields to register results of the quarter-level follow-up evaluation. Farm personnel were instructed to respect the order of the envelopes, so that each new case would correspond to the next envelope on the stack. Envelopes were opened upon inclusion of cases in the study - at confirmation of an OFC-negative result.

\section{Follow-Up Sampling and Evaluations}

Milking technicians used a standardized form to assess clinical cure and case severity, and to record possible interventions (e.g., need for antimicrobial therapy) at all milkings for $7 \mathrm{~d}$ after inclusion in the study. These evaluations were not performed during the night shift because of logistic difficulties. In addition to milk samples collected at clinical mastitis detection (d 0), additional samples were collected 14 and $21 \mathrm{~d}$ later for microbiological examination and SCC. The occurrence of clinical mastitis cases and other zootechnical data were recorded for further analysis.

\section{Study Outcomes}

Study outcomes were defined at the quarter level, as follows: clinical cure was defined as the disappearance of clinical signs within $7 \mathrm{~d}$ after inclusion in the study (yes $=1$ and no $=0$ ); relapse of clinical mastitis was defined as clinical cure was followed by observation of clinical mastitis within $14 \mathrm{~d}$ after inclusion in the study (yes $=1$ and no $=0$ ); recurrence of clinical mastitis was defined as clinical cure followed by observation of clinical mastitis 15 to $90 \mathrm{~d}$ after inclusion in the study (yes $=1$ and no $=0$ ); risk of new IMI at 14 or $21 \mathrm{~d}$ was defined as isolation of mastitis pathogens at 14 or $21 \mathrm{~d}$ from OFC-negative quarters at $0 \mathrm{~d}$ (yes $=1$ and no $=0)$; and quarter milk SCC $\left(\log _{10}\right.$ cells $\left./ \mathrm{mL}\right)$ at 14 and $21 \mathrm{~d}$.

\section{Laboratory Methods}

Microbiological analyses were performed according to the recommendations of the National Mastitis Council (1999). The investigators were blinded to the experimental groups of the milk samples. Briefly, $10 \mu \mathrm{L}$ of milk was inoculated onto blood agar and MacConkey plates and incubated for up to $72 \mathrm{~h}$ at $37^{\circ} \mathrm{C}$. An IMI was defined as growth of more than 2 similar colonies on the plate. Samples were considered contaminated when growth of more than 2 different colonies was observed on the plate. Significant growth of 2 species was defined as a mixed infection. Phenotypic microbiological diagnosis was performed based on colony morphology, Gram stain, and additional biochemical tests. Gramnegative pathogens were cultured in specific media for species-level diagnosis, such as citrate, triple sugar iron, and motility-indole-ornithine agar slants. Staphylococcus aureus was differentiated from other staphylococci by means of mannitol, trehalose, and maltose reactions, and the coagulase test. Streptococcus spp. were differentiated with the Christie, Atkins, Munch-Petersen (CAMP) test, esculin, and bile-esculin reactions.

Somatic cell counting was performed using Somaticell (IDEXX, Sao Paulo, SP, Brazil). The researcher who performed SCC was blinded to the experimental group of the sample. 


\section{Statistical Analysis}

The unit of analysis was the mammary quarter. Moderate cases $(\mathrm{n}=6)$ were excluded from analysis. Initially, we produced descriptive statistics to characterize the farms and cows included in the study. Cox proportional hazards models (PROC PHREG; SAS Institute, Cary, NC) were constructed to compare time to clinical cure, time to relapse, and time to recurrence of clinical mastitis between the groups. We performed preliminary bivariate analyses to identify explanatory variables associated with each outcome (at $P<0.10$ ). Explanatory variables considered were as follows: parity $(1,2$, and $>2$ lactations); DIM $(<100,100-199$, and $\geq 200 \mathrm{~d}$ ); quarter position (right rear, left rear, right front, and left front); and group (KET or CON).

Then, we performed a backward variable selection procedure to select final multivariable models. Because no explanatory variables were associated with any of the study outcomes, group was forced into the final models. Farm was included in the models as a random effect to account for the correlation among quarters within the same herd. Because only 2 cows had more than 1 quarter included in the study, clustering of quarters within cows was not considered.

For clinical cure, quarters were censored when clinical cure was not observed until $7 \mathrm{~d}$ after inclusion, or when quarters were removed from the study during follow-up (e.g., death, dry-off or received antimicrobial treatment). For relapse and recurrence of clinical mastitis, quarters were censored when no relapse or recurrence was observed within $14 \mathrm{~d}$ and 15 to $90 \mathrm{~d}$ after inclusion in the study, respectively, or when they were removed from the study. The assumption of proportional hazards was verified using $\log -\log$ survival curves [-ln $(-\ln$ $S)$ ], in which $S$ was the survival function.

We used generalized linear models (logistic regression; PROC GLIMMIX; SAS Institute) to estimate the odds of a new IMI at 14 and $21 \mathrm{~d}$ between groups. We used a repeated-measures mixed model (PROC MIXED; SAS Institute) to compare mean $\log _{10}$ SCC in milk samples collected at 14 and $21 \mathrm{~d}$ between groups. For both models, farm was also included as a random effect, and multivariate analyses were performed as previously described. Except for bivariate analyses, statistical significance was declared at $P<0.05$.

\section{Sample Size Calculation}

The sample size was calculated based on a superiority test (Dohoo et al., 2010) to demonstrate that KET quarters would experience a greater proportion of clinical cure than CON quarters. Calculations were per- formed assuming $\alpha$ level $=0.05$ and statistical power $=$ 0.8 . Because we found no previous negative-controlled studies investigating the efficacy of NSAID for the treatment of spontaneous, culture-negative clinical mastitis, we used a range of plausible differences in clinical cure risk between the groups $(\Delta)$ for sample size estimation. We assumed that $\Delta$ would be 0.15 (risk of clinical cure $=0.80$ and 0.95 ) and 0.20 (risk of clinical cure $=0.75$ and 0.95 ) for CON and KET, respectively. Based on these assumptions, 39 to 60 quarters per group would be required for the study. Calculations were performed using PROC POWER (SAS Institute).

\section{RESULTS}

The study was conducted between January 2018 and May 2019. Farm A participated throughout the entire study period, farm B participated in January and February 2018, and farm C participated between November 2018 and May 2019. Cows were milked 3 times/d and were confined in sand-bedded free stalls (farms B and C) or in a compost barn bedded with wood shavings (farm A). Farms A, B, and C had 285, 890, and 2,020 lactating cows that produced 37,31 , and $38.3 \mathrm{~kg} / \mathrm{cow}$ per day, respectively. Geometric mean bulk tank milk SCC, including 3 monthly tests before study onset, was 203,000, 109,000, and 165,000 cells/mL for farms A, B, and $\mathrm{C}$, respectively. The distribution of parity by farm was as follows: farm A, first lactation $=97(34.03 \%)$ and $\geq 2$ lactations $=188(65.97 \%)$ cows; Farm B, first lactation $=287(32.24 \%)$ and $\geq 2$ lactations $=603$ $(67.76 \%)$ cows; farm C, first lactation $=906(44.85 \%)$ and $\geq 2$ lactations $=1,114(55.15 \%)$ cows.

A total of 129 clinical mastitis cases from 127 cows met the inclusion criteria and were randomized into CON $(\mathrm{n}=64)$ or KET $(\mathrm{n}=65$; Figure 1$)$. After exclusion of the few moderate cases $(\mathrm{n}=6 ; 5$ cases from farm A and 1 case from farm B), 39 (31.71\%), 4 (3.25\%), and $80(65.04 \%)$ quarters remained for analysis from farms $\mathrm{A}, \mathrm{B}$, and $\mathrm{C}$, respectively. The distribution of quarters into the experimental groups was as follows: farm A, $\mathrm{KET}=20(51.28 \%)$ and $\mathrm{CON}=19(48.72 \%)$; farm B, $\mathrm{KET}=2(50.00 \%)$ and $\mathrm{CON}=2(50.00 \%)$; farm $\mathrm{C}$, $\mathrm{KET}=43(53.75 \%)$ and $\mathrm{CON}=37(46.25 \%)$. Numbers and reasons for missing and censored data relative to each outcome are presented in Figure 1.

\section{Characteristics of Experimental Groups After Randomization}

We found no significant differences between the groups after randomization for prognostic factors such as quarter position and DIM at clinical mastitis (Table 


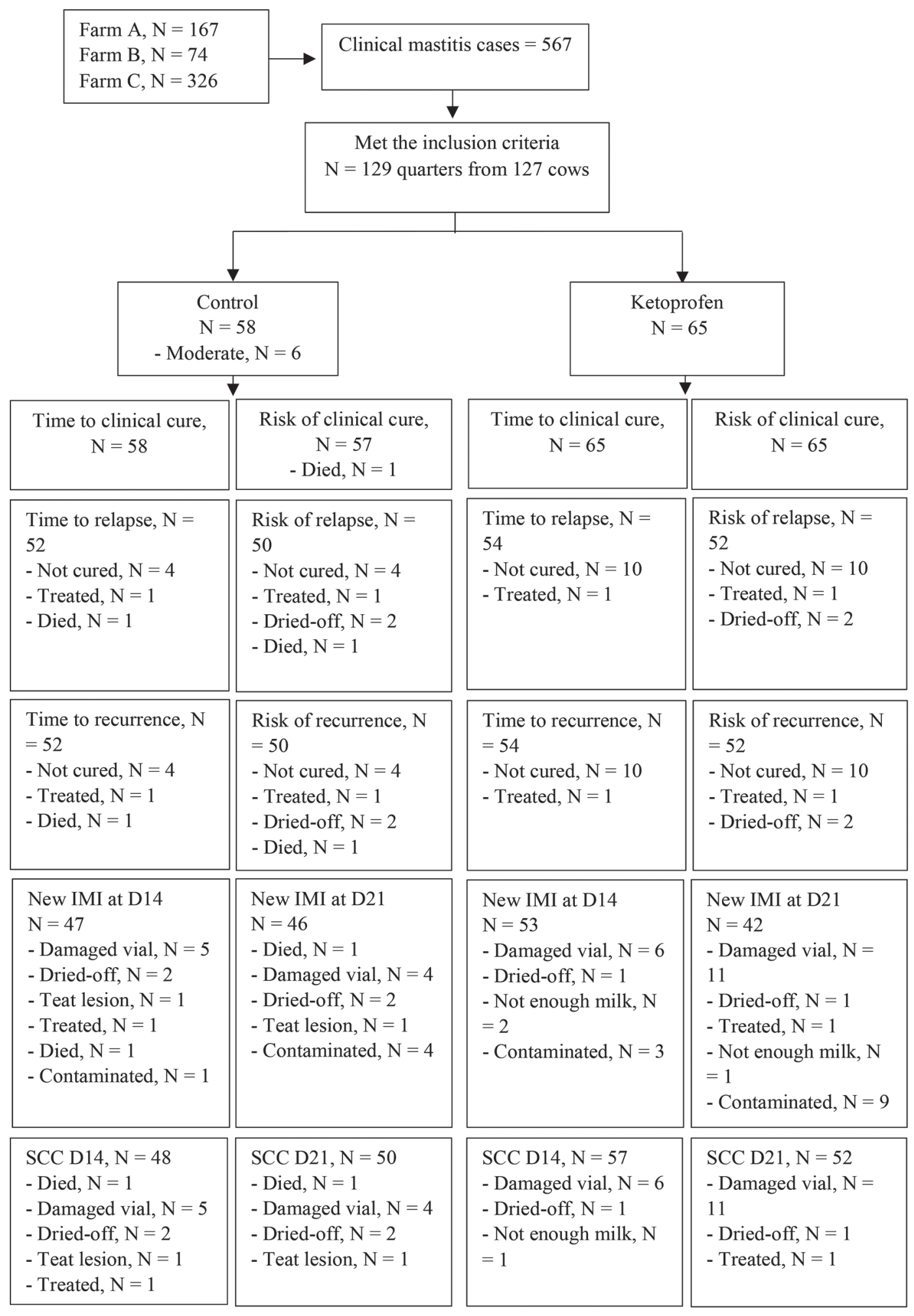

Figure 1. Completeness of the data used for analysis of each study outcome. KET: cows were treated with a single intramuscular injection of ketoprofen $(3 \mathrm{mg} / \mathrm{kg}$ of BW); CON: cows received no treatment or placebo. Clinical cure: disappearance of clinical signs within 7 d after inclusion in the study. Relapse of clinical mastitis: occurrence of clinical cure followed by observation of clinical signs within $14 \mathrm{~d}$ after inclusion in the study. Recurrence of clinical mastitis: occurrence of clinical cure followed by observation of clinical signs 15 to $90 \mathrm{~d}$ after inclusion in the study. New IMI at d 14 and 21: quarters that were culture-negative at d 0 and culture-positive 14 and $21 \mathrm{~d}$ after detection of clinical mastitis. SCC at d 14 and 21: quarter milk SCC $\left(\log _{10}\right.$ cells $\left./ \mathrm{mL}\right)$, for samples collected 14 and $21 \mathrm{~d}$ after detection of clinical mastitis. 
Table 1. Baseline characteristics of cows, mammary quarters, and clinical mastitis cases after randomization

\begin{tabular}{|c|c|c|c|}
\hline \multirow[b]{2}{*}{ Characteristic } & \multicolumn{2}{|c|}{ Group $^{1}$} & \multirow[b]{2}{*}{$P$-value ${ }^{2}$} \\
\hline & KET & $\mathrm{CON}$ & \\
\hline Quarters included in the study, no. & 65 & 58 & \\
\hline Quarter position, \% (no./total) & & & 0.98 \\
\hline Right front & $35.38(23 / 65)$ & $36.21(21 / 58)$ & \\
\hline Left front & $29.23(19 / 65)$ & $25.86(15 / 58)$ & \\
\hline Right rear & $10.77(7 / 65)$ & $12.07(7 / 58)$ & \\
\hline Left rear & $24.62(16 / 65)$ & $25.86(15 / 58)$ & \\
\hline Parity $^{3}$ & $2.00(2.00-3.00)$ & $3.00(2.00-3.00)$ & 0.04 \\
\hline DIM at $\mathrm{d} 0^{3,4}$ & $137.00(75.00-228.00)$ & $111.00(72.00-213.00)$ & 0.68 \\
\hline Interval $\mathrm{d} 0$ to $14, \mathrm{~d}^{3,5}$ & $14.00(13.00-16.00)$ & $14.00(13.50-16.00)$ & 0.87 \\
\hline Interval $\mathrm{d} 0$ to $21, \mathrm{~d}^{3,5}$ & $21.00(20.00-23.00)$ & $21.00(21.00-24.00)$ & 0.89 \\
\hline \multicolumn{4}{|c|}{$\begin{array}{l}{ }^{1} \mathrm{KET}=\text { cows were treated with a single intramuscular injection of ketoprofen }(3 \mathrm{mg} / \mathrm{kg} \text { of } \mathrm{BW}) ; \mathrm{CON}=\text { cows } \\
\text { received no treatment or placebo. }\end{array}$} \\
\hline \multirow{2}{*}{\multicolumn{4}{|c|}{$\begin{array}{l}{ }^{2} \text { Wilcoxon rank-sum test for continuous variables and } \chi^{2} \text { test (or Fisher's exact test) for categorical variables. } \\
{ }^{3} \text { Findings presented as median (1st quartile to } 3 \text { rd quartile). } \\
{ }^{4} \text { DIM at clinical mastitis detection. }\end{array}$}} \\
\hline & & & \\
\hline \multicolumn{4}{|c|}{$\begin{array}{l}{ }^{5} \text { Interval between clinical mastitis detection and milk sampling ( } 14 \text { and } 21 \mathrm{~d} \text { post-detection) for microbiological } \\
\text { examination and SCC. }\end{array}$} \\
\hline
\end{tabular}

1). We did find a median difference of 1 lactation between the groups. For both groups, the median intervals between clinical mastitis and 14 or $21 \mathrm{~d}$ sampling were 14 and $21 \mathrm{~d}$, respectively (Table 1 ).

At $0 \mathrm{~d}, 10.34 \%(6 / 58)$ of the OFC-negative cases allocated to CON were culture-positive at the research laboratory (i.e., false-negative OFC result). Pathogens isolated from these samples were Klebsiella pneumoniae $(\mathrm{n}=3)$, CNS $(\mathrm{n}=2)$, and Corynebacterium bovis ( $\mathrm{n}$ $=1)$.

At $0 \mathrm{~d}, 10.77 \%(7 / 65)$ of the OFC-negative cases allocated to KET were culture-positive at the research laboratory. Pathogens isolated from these samples were C. bovis $(\mathrm{n}=2)$, CNS $(\mathrm{n}=2)$, Enterococcus $\mathrm{spp}$. $(\mathrm{n}=$ $1)$, Streptococcus uberis $(\mathrm{n}=1)$, and yeast $(\mathrm{n}=1)$. The percentage of OFC false-negative culture results at $0 \mathrm{~d}$ was not different between the groups $(P=0.71)$.

\section{Clinical Cure}

None of the explanatory variables was associated with time to clinical cure within $7 \mathrm{~d}$ after inclusion in the study. Therefore, group was forced into the final model and farm remained as a random term. The risk of clinical cure was $83.08 \%(54 / 65)$ and $91.23 \%(52 / 57)$ for KET and CON, respectively (Table 2), and was not different between groups [hazard ratio $(\mathbf{H R})=1.20$; 95\% CI $=0.82-1.76 ; P=0.35$; Figure 2 and Table 3].

\section{Relapse and Recurrence of Clinical Mastitis}

None of the explanatory variables was associated with time to relapse or recurrence of clinical mastitis.
Group was forced into the final model, and farm remained as a random term (Table 3). Risk of relapse was $19.23 \%(10 / 52)$ and $18.00 \%(9 / 50)$ for KET and CON, respectively (Table 2), and was not different between the groups ( $\mathrm{HR}=1.09 ; 95 \% \mathrm{CI}: 0.45-2.62 ; P=0.85$; Figure 3 and Table 3 ).

Risk of recurrence was $17.31 \%(9 / 52)$ and $18.00 \%$ $(9 / 50)$ for KET and CON, respectively (Table 2), and was not different between the groups ( $\mathrm{HR}=1.26 ; 95 \%$ CI: $0.49-3.38 ; P=0.61$; Figure 3 and Table 3 ).

\section{Risk of New IMI}

At $14 \mathrm{~d}, 20.75 \%(11 / 53)$ and $29.79 \%(14 / 47)$ of KET and CON quarters developed new IMI, respectively (Table 2). None of the explanatory variables was associated with the chances of new IMI at $14 \mathrm{~d}$. Group was forced into the final logistic regression model, and farm was included as a random term (Table 3). The odds of new IMI at $14 \mathrm{~d}$ were not different between the groups [odds ratio $(\mathbf{O R})=1.76 ; 95 \%$ CI: $0.66-4.73 ; P$ $=0.26$; Table 3].

For KET quarters, new IMI at $14 \mathrm{~d}$ were caused by Streptococcus spp. $(\mathrm{n}=4)$, CNS $(\mathrm{n}=5)$, C. bovis $(\mathrm{n}=$ $2)$, yeast $(\mathrm{n}=1)$, and Bacillus spp. $(\mathrm{n}=1)$. For CON quarters, new IMI were caused by CNS $(\mathrm{n}=5)$, Bacillus spp. $(\mathrm{n}=2)$, C. bovis $(\mathrm{n}=2)$, Streptococcus spp. (n $=2), K$. pneumoniae $(\mathrm{n}=1)$, Klebsiella oxytoca $(\mathrm{n}=$ $1)$, and mixed infection $(\mathrm{n}=1)$.

At $21 \mathrm{~d}, 28.57 \%(12 / 42)$ and $15.22 \%(7 / 46)$ of KET and CON quarters developed new IMI, respectively (Table 2). None of the explanatory variables was associated with the chances of new IMI at $21 \mathrm{~d}$. Group was 


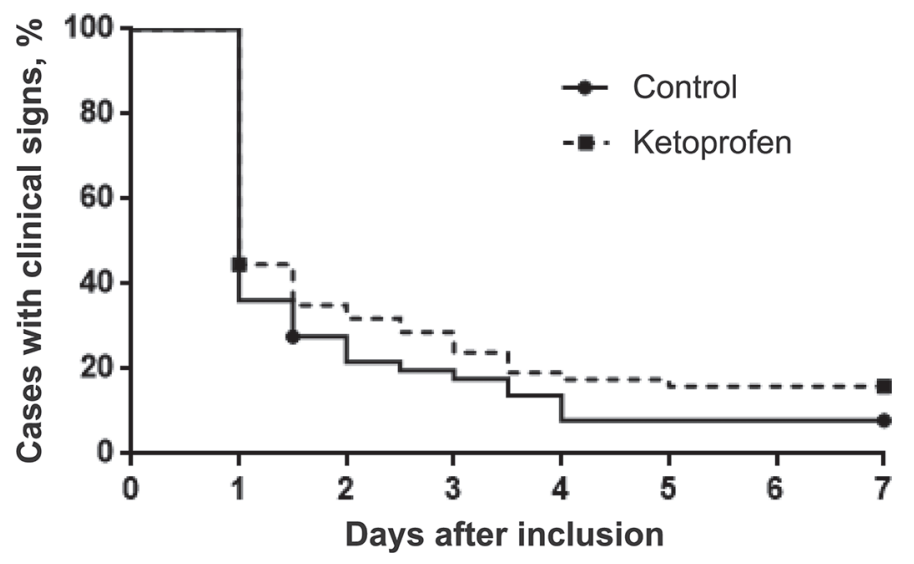

Figure 2. Survival curve depicting time to clinical cure within $7 \mathrm{~d}$ after inclusion in the study. Cases were included in the study approximately $24 \mathrm{~h}$ after detection, upon confirmation of a culture-negative result. Ketoprofen $=$ cows were treated with a single intramuscular injection of ketoprofen $(3 \mathrm{mg} / \mathrm{kg}$ of BW $)$; control = cows received no treatment or placebo. Clinical cure: disappearance of clinical signs within $7 \mathrm{~d}$ after inclusion in the study.

forced into the final logistic regression model, and farm was included as a random term (Table 3 ). The odds of new IMI at $21 \mathrm{~d}$ were not different between the groups $(\mathrm{OR}=0.45 ; 95 \%$ CI: $0.16-1.30 ; P=0.14$; Table 3$)$.

For KET quarters, new IMI at $21 \mathrm{~d}$ were caused by Streptococcus spp. $(\mathrm{n}=5)$, CNS $(\mathrm{n}=2), C$. bovis ( $=1)$, Enterococcus spp. $(\mathrm{n}=1)$, Escherichia coli $(\mathrm{n}=$ $1), K$. oxytoca $(\mathrm{n}=1)$, and Pseudomonas spp. $(\mathrm{n}=1)$. For CON quarters, new IMI were caused by coagulase- negative staphylococci $(\mathrm{n}=3)$, Bacillus spp. $(\mathrm{n}=2)$, K. oxytoca $(\mathrm{n}=1)$, and Prototheca spp. $(\mathrm{n}=1)$.

\section{SCC}

Mean SCC was not different between the groups at $14 \mathrm{~d}$ or $21 \mathrm{~d}$ (Table 3 and Figure 4).

\section{DISCUSSION}

Farmers and veterinary practitioners have been increasingly using ketoprofen for the treatment of OFCnegative clinical mastitis cases in an attempt to reduce time to clinical cure and days of milk discard. This practice motivated us to test the hypothesis that treatment of such cases with a single intramuscular injection of ketoprofen would reduce time to clinical cure, relapse, and recurrence of clinical mastitis, decrease risk of subsequent IMI, and lower milk SCC compared with untreated controls.

To our knowledge, the distribution of ketoprofen in the mastitic mammary gland and its elimination in milk after intramuscular or intravenous administration have not been reported. For healthy cows, Daeseleire et al. (2003) used liquid chromatography-tandem mass spectrometry to determine milk concentrations of ketoprofen after intravenous administration and reported that the drug could be detected in milk in very low concentrations for a short period of time $(<10 \mathrm{~h})$.

Table 2. Descriptive statistics for study outcomes by experimental group

\begin{tabular}{|c|c|c|}
\hline \multirow[b]{2}{*}{ Variable $^{1}$} & \multicolumn{2}{|c|}{ Group $^{2}$} \\
\hline & KET & $\mathrm{CON}$ \\
\hline
\end{tabular}

\footnotetext{
${ }^{1}$ Numbers and reasons for missing data are presented in Figure 1.

${ }^{2} \mathrm{KET}=$ cows were treated with a single intramuscular injection of ketoprofen $(3 \mathrm{mg} / \mathrm{kg}$ of BW $)$; CON $=$ cows received no treatment or placebo. ${ }^{3}$ Disappearance of clinical signs within $7 \mathrm{~d}$ after inclusion in the study. Cases were included in the study approximately $24 \mathrm{~h}$ post-detection, upon confirmation of a culture-negative result.

${ }^{4}$ Occurrence of clinical cure followed by observation of clinical mastitis within $14 \mathrm{~d}$ after inclusion in the study.

${ }^{5}$ Occurrence of clinical cure followed by observation of clinical mastitis 15 to $90 \mathrm{~d}$ after inclusion in the study.

${ }^{6}$ Quarters that were culture-negative at d 0 (clinical mastitis detection) and culture-positive at d 14 (14 d post-detection).

${ }^{7}$ Quarters that were culture-negative at d 0 and culture-positive at d 21 (21 d post-detection).

${ }^{8}$ Findings presented as median (1st quartile to 3rd quartile).

${ }^{9}$ Findings presented as mean \pm standard error of the mean.
} 
Table 3. Multivariate analysis to compare study outcomes between experimental groups

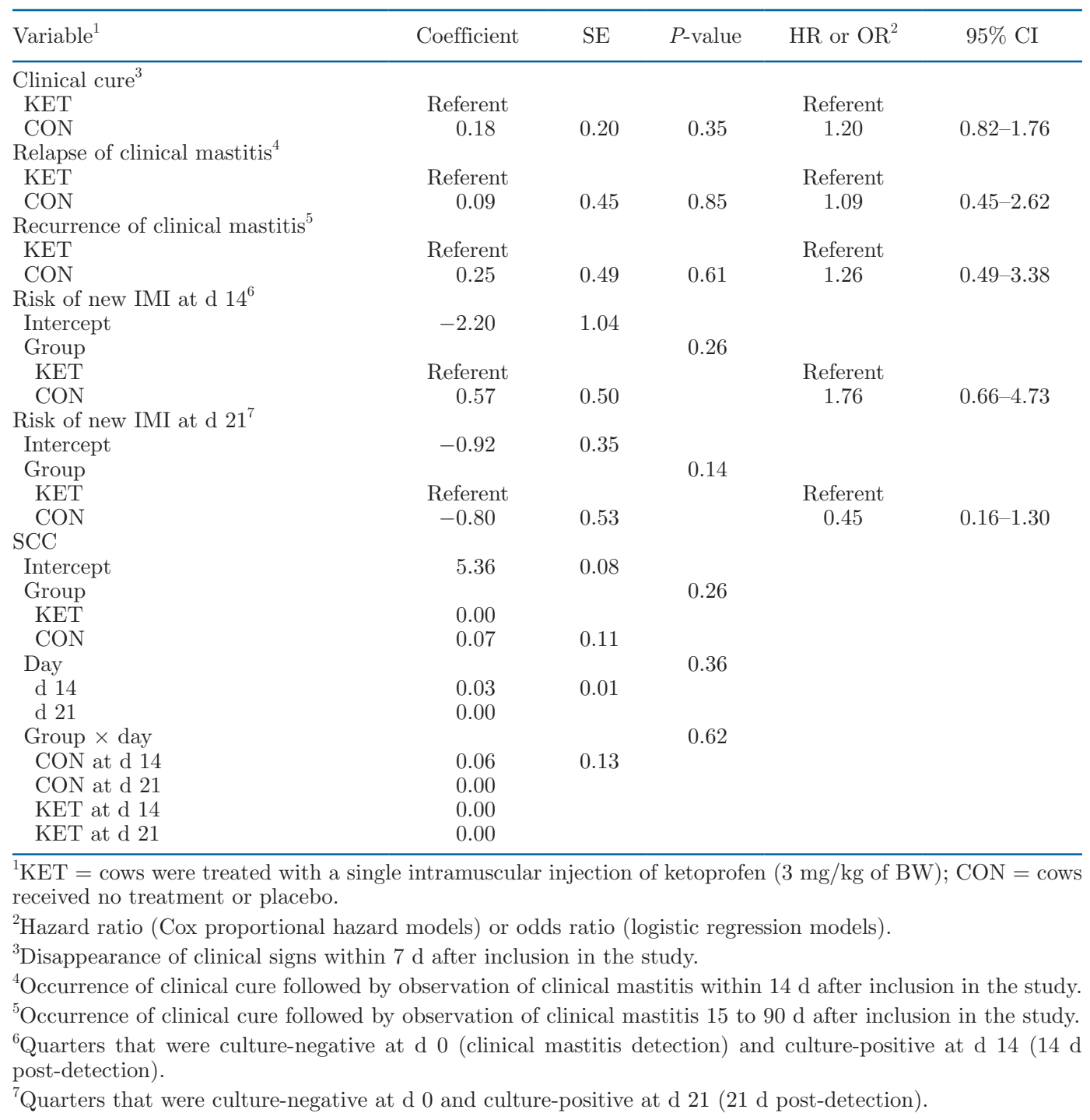

Nonetheless, the efficacy of ketoprofen for treating spontaneous and experimental clinical mastitis has been previously reported. Shpigel et al. (1994) reported that spontaneous clinical mastitis treated intramuscularly with $2 \mathrm{~g}$ of ketoprofen once per day, in addition to sulphadiazine and trimethoprim, had an odds of recovery (return to $>75 \%$ of the pre-mastitis daily milk production) 6.8 times greater than controls. Zecconi et al. (2018) treated chronically infected cows (defined as $\mathrm{SCC}>400,000$ cells $/ \mathrm{mL}$ in the last 2 monthly test days) intramuscularly with ketoprofen $(3 \mathrm{mg} / \mathrm{kg})$ and observed significant reductions in the occurrence of abnormal milk flow curves and milk electrical conductivity. In addition, significant reductions in udder size and concentration of thromboxane $\mathrm{B}_{2}$ were found in the milk of cows experimentally infected with $E$. coli and treated intramuscularly with ketoprofen (3 mg/ $\mathrm{kg}$ ), compared with untreated controls (Banting et al., 2008). The results of these studies demonstrate a reduction in inflammation in the mammary gland after intramuscular treatment with ketoprofen.

Other studies that investigated the effect of NSAID to treat spontaneous clinical mastitis used meloxicam as the drug of choice and suggested some beneficial effects of a single intramuscular injection, concomitant to intramammary antimicrobials, on improvement in reproductive performance, remission of inflammatory signs, and SCC decrease after treatment (McDougall et al., 2009; McDougall et al., 2016). The effect of NSAID on the microbiological cure of clinical mastitis has not been well studied. Although one study did not report a significant effect (McDougall et al., 2009), a more 

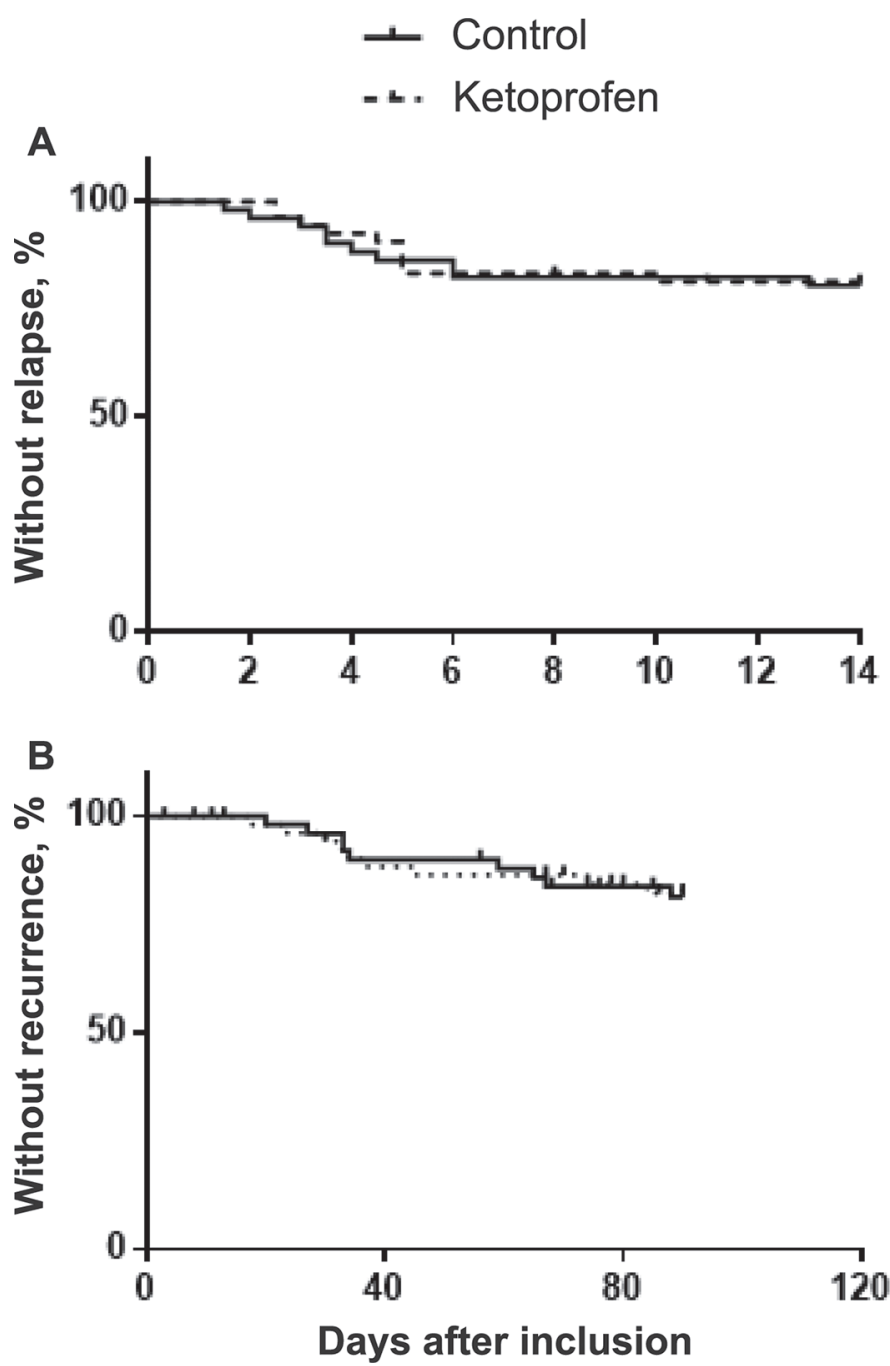

Figure 3. Survival curves depicting time to relapse (A) or recurrence (B) of clinical mastitis. Relapse was defined as occurrence of clinical cure followed by observation of clinical signs within $14 \mathrm{~d}$ after inclusion in the study. Recurrence was defined as occurrence of clinical cure followed by observation of clinical signs 15 to $90 \mathrm{~d}$ after inclusion in the study. Cases were included in the study approximately $24 \mathrm{~h}$ post-detection, upon confirmation of a culture-negative result. Ketoprofen $=$ cows were treated with a single intramuscular injection of ketoprofen $(3 \mathrm{mg} / \mathrm{kg}$ of $\mathrm{BW})$; control = cows received no treatment or placebo.

recent trial reported an increase of 16 percentage points in microbiological cure risk for quarters treated with intramammary cefalexin and kanamycin associated with a single intramuscular injection of meloxicam (cure risk $=66 \%$ ), compared with quarters treated with only the antimicrobials (cure risk $=50 \%$; McDougall et al., 2016).

The results of the present study did not support the use of ketoprofen for the treatment of OFC-negative, mild clinical mastitis cases based on the outcomes we studied. As part of the inflammatory process, a mas-

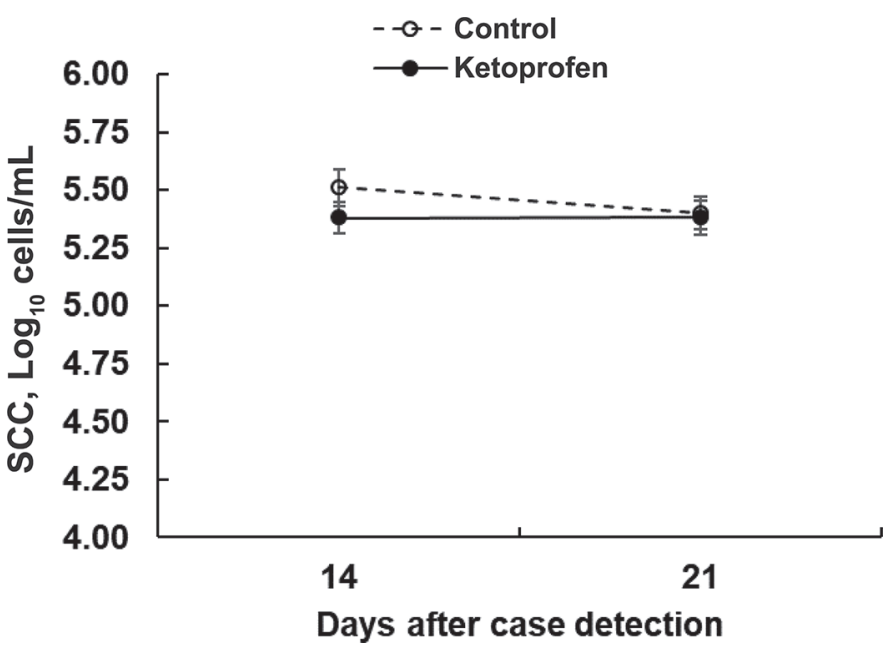

Figure 4. Quarter milk SCC $\left(\log _{10}\right.$ cells $/ \mathrm{mL}$; geometric mean and $95 \%$ confidence interval) at 14 and $21 \mathrm{~d}$ after detection of clinical mastitis. Ketoprofen $=$ cows were treated with a single intramuscular injection of ketoprofen $(3 \mathrm{mg} / \mathrm{kg}$ of BW $)$; control = cows received no treatment or placebo.

sive influx of leukocytes from the bloodstream into the mammary gland occurs to destroy the invading pathogens. The release of substances such as proinflammatory cytokines, oxygen radicals, peroxidases, and proteases (Aitken, et al., 2011) occurs as a result of the damage caused by pathogens and the phagocytic action and apoptosis of leukocytes and epithelial cells. As a result, alveolar tissue and milk components are destroyed, compromising the quantity and quality of milk (Sordillo et al., 1997). Clinical mastitis treatment with ketoprofen could result in attenuation of these deleterious effects caused by the inflammatory process in the mammary gland tissues and milk.

However, considering the time between infection and the detection of clinical signs (approximately 18 h; Sipka et al., 2013) and additional OFC (24 h), cows were treated at least $40 \mathrm{~h}$ from infection onset, at which point the inflammatory process is expected to be fully established in the mammary gland (Sipka et al., 2013). We observed an unexpected data trend of shorter time to clinical cure (Figure 2) for CON quarters, suggesting that the use of ketoprofen at that point might have a negative effect on time to clinical cure. This hypothesis may be partially explained by the suppression of cells and mediators that are responsible for resolution of inflammation and mammary tissue repair, such as oxylipids and cytokines (Sordillo, 2018). Dan et al. (2018) studied the effect of intramammary ketoprofen during the lipopolysaccharide-induced immune response in mammary glands and demonstrated, in vivo and in vitro, the suppression of SCC increase and the reduction of damage to the blood-milk barrier (based on the 
concentration of serum albumin and lactate dehydrogenase), reduction of IgG concentration, and expression of various inflammatory factors in milk during the inflammatory process. In agreement with previous studies (McDougall et al., 2009; Dan et al., 2018), we observed a greater numerical decrease in mean SCC (not statistically significant) for KET quarters at $14 \mathrm{~d}$ compared with CON. Although not statistically significant, these trends could be worthy of further investigation.

Time to clinical mastitis relapse, recurrence, and risk of new IMI were also not different between the groups in the present study. Swartz et al. (2018) observed that cows treated with meloxicam during the peripartum period had lower incidence of clinical mastitis than untreated controls, suggesting that NSAID could have a preventive effect on clinical mastitis. Nonetheless, it was difficult to extrapolate the results of that study to other stages of lactation, because of great differences in immune response competency.

Time to clinical cure was similar to those reported in previous studies. Lago et al. (2011a) and Fuenzalida and Ruegg (2019) conducted trials including OFCnegative, untreated clinical mastitis cases and reported that mean days to clinical cure after detection were 3 and 4 , respectively. In the present study, the median days to clinical cure after study inclusion was 1 for CON and KET, but the means were 2.0 and $2.4 \mathrm{~d}$, respectively, justifying the use of the median. Considering that most cases were included $24 \mathrm{~h}$ after detection (upon confirmation of culture-negative results), times to clinical cure after detection were approximately 2 and $2 \mathrm{~d}$ (median) and 3 and $3.4 \mathrm{~d}$ (mean) for CON and KET, respectively.

Seven $(10.77 \%)$ and $6(10.34 \%)$ KET and CON OFC-negative samples at $0 \mathrm{~d}$, respectively, were culture-positive according to the research laboratory (i.e., false-negative results). These samples were not excluded from the analyses because the goal of the study was to investigate the efficacy of ketoprofen for treatment of culture-negative cases diagnosed with actual OFC systems, which may not be as sensitive for detecting mastitis pathogens (Griffioen et al., 2018). The objective of comparing OFC and research laboratory results was to verify the possibility of bias, which could have been introduced if the distribution of false-negative OFC results was different between the groups. Because the distribution of false-negative results was homogeneous between the groups, it was unlikely that bias was introduced. The occurrence of false-negative results can be explained by differences in culture methodology, and other factors such as shipping temperature and freezing of milk samples. Differences in incubation time $(24 \mathrm{~h}$ on the farm and $72 \mathrm{~h}$ in the research laboratory), allow for the isolation of slow-growing pathogens such as
Corynebacterium spp., Prototheca spp., Nocardia spp., and Trueperella pyogenes (Markey et al., 2013). This fact highlights the importance of submitting all clinical mastitis samples to reference laboratories (regardless of OFC results) to avoid misdiagnosis of relevant pathogens.

We believe that the results of this study can be extrapolated to herds composed of high-producing Holsteins housed in confinement systems that experience mild clinical mastitis.

\section{Study Limitations}

One of the limitations of this study was the lack of clinical mastitis evaluation (clinical cure) during the night shift. All participant farm managers mentioned that it would be difficult for milking technicians to fill out the study form during the night shift, because of lower staff levels. This may have resulted in overestimation of time to clinical cure, because cases that were clinically cured during the night shift were evaluated the next morning. However, it is unlikely that bias was introduced, because overestimation of time to clinical cure was probably randomly distributed between the groups.

The time interval between case detection and inclusion in the study (upon completion of a culture-negative diagnosis) was not precisely recorded. Although the farms had a steady culturing routine (e.g., culturing was performed daily at the same times), cases from farms $\mathrm{A}$ and $\mathrm{B}$ that were detected during the morning or afternoon shift were cultured right away, and cases detected at night were cultured the next morning. On farm $\mathrm{C}$, cases detected during the afternoon or night shift were cultured the next morning, and cases detected during the morning shift were cultured right away. We used the moment of inclusion in the study (which was also the moment of treatment for KET quarters) as the starting point for the follow-up period for assessment of clinical cure, relapse, and recurrence of clinical mastitis. It is unlikely that bias was introduced, because the time interval between case detection and inclusion was probably randomly distributed between the groups.

Previous studies that investigated efficacy of NSAID to treat clinical mastitis have included mild and moderate cases, but the distribution of case severity was not reported (McDougall et al., 2009, 2016). The proportion of mild and moderate clinical mastitis has been reported as 48 to $65 \%$ (Wenz et al., 2001) and 27 to 31\% (Pinzón-Sánchez and Ruegg, 2011). In contrast, the low number of moderate cases enrolled in the present study $(\mathrm{n}=6)$ was probably due to the farmers' urge to treat more severe cases before waiting for OFC results. Because moderate cases were excluded from 
the analyses, our results can be extrapolated to mild cases of clinical mastitis. Further studies are necessary to investigate the efficacy of ketoprofen for treatment of moderate clinical mastitis.

\section{CONCLUSIONS}

The results of this study suggest that a single intramuscular injection of ketoprofen as the sole treatment for OFC-negative, mild clinical mastitis did not reduce time to clinical cure, relapse, or recurrence of clinical mastitis, decrease risk of subsequent IMI, or lower milk SCC compared with untreated controls.

\section{ACKNOWLEDGMENTS}

We are grateful to the dairy farmers and their staff, and the graduate students who participated in this study, especially Fabio Lucas Resende de Gouvea e Ananda Finco de Cesar Lopes. We thank CEVA Saude Animal (Paulinia, Brazil) for donating some of the drugs used in this trial. The scholarship of Giulia S. Latosinski was funded by the Sao Paulo Research Foundation (FAPESP), Grant 2017/20576-5. The authors have not stated any conflicts of interest.

\section{REFERENCES}

Aitken, S. L., C. M. Corl, and L. M. Sordillo. 2011. Immunopathology of mastitis: Insights into disease recognition and resolution. J. Mammary Gland Biol. Neoplasia 16:291-304. https://doi.org/10 .1007/s10911-011-9230-4.

Banting, A., S. Banting, K. Heinonen, and K. Mustonen. 2008. Efficacy of oral and parenteral ketoprofen in lactating cows with endotoxin-induced acute mastitis. Vet. Rec. 163:506-509. https:// doi.org/10.1136/vr.163.17.506.

Cardoso, C. S., M. J. Hotzel, D. M. Weary, J. A. Robbins, and M. A. G. Von Keyserlingk. 2016. Imagining the ideal dairy farm. J. Dairy Sci. 99:1663-1671. https://doi.org/10.3168/jds.2015-9925.

Cortinhas, C. S., T. Tomazi, M. S. F. Zoni, E. Moro, and M. Veiga dos Santos. 2016. Randomized clinical trial comparing ceftiofur hydrochloride with a positive control protocol for intramammary treatment of nonsevere clinical mastitis in dairy cows. J. Dairy Sci. 99:5619-5628. https://doi.org/10.3168/jds.2016-10891.

Daeseleire, E., L. Mortier, H. De Ruyck, and N. Geerts. 2003. Determination of flunixin and ketoprofen in milk by liquid chromatography - tandem mass spectrometry. Anal. Chim. Acta 488:25-34. https://doi.org/10.1016/S0003-2670(03)00577-4.

Dan, D., R. M. Bruckmaier, and O. Wellnitz. 2018. Ketoprofen affects the mammary immune response in dairy cows in vivo and in vitro. J. Dairy Sci. 101:11321-11329. https://doi.org/10.3168/jds .2018-15034.

Dohoo, I., S. W. Martin, and H. Stryhn. 2010. Veterinary Epidemiologic Research. 2nd ed. VER Inc., Charlottetown, PE, Canada.

Friedman, L. M., C. Furberg, D. L. Demets, D. M. Reboussin, and C. B. Granger. 2010. Fundamentals of Clinical trials. 4th ed. Springer International Publishing, New York, NY.

Fuenzalida, M. J., and P. L. Ruegg. 2019. Negatively controlled, randomized clinical trial to evaluate use of intramammary ceftiofur for treatment of nonsevere culture-negative clinical mastitis. J. Dairy Sci. 102:3321-3338. https://doi.org/10.3168/jds.2018-15497.
Griffioen, K., A. G. J. Velthuis, L. A. Lagerwerf, A. E. Heuvelink, and T. J. G. M. Lam. 2018. Agreement between four commercial diagnostic tests and routine bacteriological culture of milk to determine the udder infection status of dairy cows. Prev. Vet. Med. 157:162-173. https://doi.org/10.1016/j.prevetmed.2018.07.003.

Halasa, T., K. Huijps, O. Osteras, and H. Hogeveen. 2007. Economic effects of bovine mastitis and mastitis management: A review. Vet. Q. 29:18-31. https://doi.org/10.1080/01652176.2007.9695224.

Kantor, T. G. 1986. A review of its pharmacologic and clinical properties. Pharmacotherapy 6:93-102. https://doi.org/10.1002/j.1875 -9114.1986.tb03459.x.

Kayitsinga, J., R. L. Schewe, G. A. Contreras, and R. J. Erskine. 2017. Antimicrobial treatment of clinical mastitis in the eastern United States: The influence of dairy farmers' mastitis management and treatment behavior and attitudes. J. Dairy Sci. 100:1388-1407. https://doi.org/10.3168/jds.2016-11708.

Lago, A., S. M. Godden, R. Bey, P. L. Ruegg, and K. Leslie. 2011a. The selective treatment of clinical mastitis based on on-farm culture results: I. Effects on antibiotic use, milk withholding time, and short-term clinical and bacteriological outcomes. J. Dairy Sci. 94:4441-4456. https://doi.org/10.3168/jds.2010-4046.

Lago, A., S. M. Godden, R. Bey, P. L. Ruegg, and K. Leslie. 2011b. The selective treatment of clinical mastitis based on on-farm culture results: II. Effects on lactation performance, including clinical mastitis recurrence, somatic cell count, milk production, and cow survival. J. Dairy Sci. 94:4457-4467. https://doi.org/10.3168/jds .2010-4047.

Markey, B., F. Leonard, M. Archambault, A. Cullinane, and D. Maguire. 2013. Clinical Veterinary Microbiology. 2nd ed. Elsevier, Dublin, Ireland.

McDougall, S., E. Abbeloos, S. Piepers, A. S. Rao, S. Astiz, T. Van Werven, J. Statham, and N. Pérez-Villalobos. 2016. Addition of meloxicam to the treatment of clinical mastitis improves subsequent reproductive performance. J. Dairy Sci. 99:2026-2042. https: //doi.org/10.3168/jds.2015-9615.

McDougall, S., D. G. Arthur, M. A. Bryan, J. J. Vermunt, and A. M. Weir. 2007. Clinical and bacteriological response to treatment of clinical mastitis with one of three intramammary antibiotics. N. Z. Vet. J. 55:161-170. https://doi.org/10.1080/00480169.2007.36762.

McDougall, S., M. A. Bryan, and R. M. Tiddy. 2009. Effect of treatment with the nonsteroidal antiinflammatory meloxicam on milk production, somatic cell count, probability of retreatment, and culling of dairy cows with mild clinical mastitis. J. Dairy Sci. 92:4421-4431. https://doi.org/10.3168/jds.2009-2284.

National Mastitis Council. 1999. Laboratory Handbook on Bovine Mastitis. Natl. Mastitis Counc., Verona, WI.

NOAH (National Office of Animal Health). 2018. Rifen $100 \mathrm{mg} / \mathrm{ml}$ solution for injection for horses, cattle and swine: Further information. Accessed Sep. 20, 2018. http://www.noahcompendium.co $. u k / ? \mathrm{id}=-449368$.

Nobrega, D. B., J. De Buck, S. A. Naqvi, G. Liu, S. Naushad, V. Saini, and H. W. Barkema. 2017. Comparison of treatment records and inventory of empty drug containers to quantify antimicrobial usage in dairy herds. J. Dairy Sci. 100:9736-9745. https://doi.org/10 $.3168 /$ jds.2017-13116.

Pinzón-Sánchez, C., and P. L. Ruegg. 2011. Risk factors associated with short-term post-treatment outcomes of clinical mastitis. J. Dairy Sci. 94:3397-3410. https://doi.org/10.3168/jds.2010-3925.

Redding, L. E., J. Bender, and L. Baker. 2019. Quantification of antibiotic use on dairy farms in Pennsylvania. J. Dairy Sci. 102:14941507. https://doi.org/10.3168/jds.2018-15224.

Sargeant, J. M., A. O'Connor, I. R. Dohoo, H. N. Erb, M. Cevallos, M. Egger, A. K. Ersbøll, S. W. Martin, L. R. Nielsen, D. L. Pearl, D. U. Pfeiffer, J. Sanchez, M. E. Torrence, H. Vigre, C. Waldner, and M. P. Ward. 2016. Methods and processes of developing the strengthening the reporting of observational studies in epidemiology - veterinary (STROBE-Vet) statement. Prev. Vet. Med. 134:188-196. https://doi.org/10.1016/j.prevetmed.2016.09.005.

Shpigel, N. Y., R. Chen, M. Winkler, A. Saran, G. Ziv, and F. Longo. 1994. The anti-inflammatory ketoprofen in the treatment of field 
cases of bovine mastitis. Res. Vet. Sci. 56:62-68. https://doi.org/ 10.1016/0034-5288(94)90197-X.

Sipka, A., A. Gurjar, S. Klaessig, G. E. Duhamel, A. Skidmore, J. Swinkels, P. Cox, and Y. Schukken. 2013. Prednisolone and cefapirin act synergistically in resolving experimental Escherichia coli mastitis. J. Dairy Sci. 96:4406-4418. https://doi.org/10.3168/jds 2012-6455.

Sordillo, L. M. 2018. Mammary gland immunobiology and resistance to mastitis. Vet. Clin. North Am. Food Anim. Pract. 34:507-523. https://doi.org/10.1016/j.cvfa.2018.07.005.

Sordillo, L. M., K. Shafer-Weaver, and D. Derosa. 1997. Immunobiology of the mammary gland. J. Dairy Sci. 80:1851-1865. https:// doi.org/10.3168/jds.S0022-0302(97)76121-6.

Swartz, T. H., H. H. Schramm, J. M. Bewley, C. M. Wood, K. E. Leslie, and C. S. Petersson-Wolfe. 2018. Meloxicam administration either prior to or after parturition: Effects on behavior, health, and production in dairy cows. J. Dairy Sci. 101:10151-10167. https:// doi.org/10.3168/jds.2018-14657.

Vane, J. R., and R. M. Botting. 1998. Mechanism of action of antiinflammatory drugs: An overview. J. Vane and J. Botting, ed. Springer, Dordrecht, the Netherlands.
Vasquez, A. K., D. V. Nydam, M. B. Capel, S. Eicker, and P. D. Virkler. 2017. Clinical outcome comparison of immediate blanket treatment versus a delayed pathogen-based treatment protocol for clinical mastitis in a New York dairy herd. J. Dairy Sci. 100:29923003. https://doi.org/10.3168/jds.2016-11614.

Wenz, J. R., G. M. Barrington, F. B. Garry, R. P. Dinsmore, and R. J. Callan. 2001. Use of systemic disease signs to assess disease severity in dairy cows with acute coliform mastitis. J. Am. Vet. Med. Assoc. 218:567-572. https://doi.org/10.2460/javma.2001.218.567.

Zecconi, A., S. Frosi, M. Cipolla, and C. Gusmara. 2018. Effects of chronic mastitis and its treatment with ketoprofen on the milk ejection curve. J. Dairy Res. 85:50-52. https://doi.org/10.1017/ S0022029917000863.

\section{ORCIDS}

J. C. F. Pantoja () https://orcid.org/0000-0002-1648-9360 